\title{
Eating high fat chow, but not drinking sucrose or saccharin, enhances the development of sensitization to the locomotor effects of cocaine in adolescent female rats
}

\author{
Katherine M. Serafine ${ }^{1}$, Todd A. Bentley ${ }^{1}$, Wouter Koek ${ }^{1,2}$, and Charles P. France ${ }^{1,2}$ \\ ${ }^{1}$ Department of Pharmacology, University of Texas Health Science, Center at San Antonio, 7703 \\ Floyd Curl Drive, Mail Code 7764, San Antonio, TX 78229 \\ ${ }^{2}$ Department of Psychiatry, University of Texas Health Science, Center at San Antonio, 7703 \\ Floyd Curl Drive, Mail Code 7764, San Antonio, TX 78229
}

\begin{abstract}
Eating high fat chow accelerates the development of sensitization to cocaine-induced locomotion in female rats. It is not known whether consumption of sucrose or saccharin also increases sensitivity to the behavioral effects of cocaine or whether continuous (or intermittent) access to these feeding conditions is necessary to change sensitivity. Adolescent female Sprague-Dawley rats were assigned to 1 of 7 feeding conditions from post natal day (PND) 25 through 60. Rats ate either high fat ( $60 \% \mathrm{kcal}$ from fat) chow and drank water, or standard (17\% kcal from fat) chow and drank either water, $10 \%$ sucrose solution, or $0.1 \%$ saccharin solution. Rats had either continuous access to high fat chow, sucrose or saccharin or they had intermittent access (i.e. 2 days per week) to these substances with access to water and standard chow on other days. As compared with rats eating standard chow, continuous (but not intermittent) access to high fat chow enhanced the development of sensitization to cocaine-induced $(1-17.8 \mathrm{mg} / \mathrm{kg}$ ) locomotion; drinking sucrose or saccharin (continuous or intermittent access) did not alter the development of sensitization to cocaine-induced locomotion. The impact of feeding condition on behavioral effects of cocaine varies between sexes and across dietary composition.
\end{abstract}

\section{Keywords}

high fat chow; sucrose; saccharin; locomotion; cocaine; sensitization; rat

\section{Introduction}

Eating highly preferred foods can impact sensitivity to the behavioral effects of drugs that act on dopamine systems (Avena and Hoebel, 2003; Baladi et al., 2012a; Gosnell 2005). For example, rats eating high fat chow are more sensitive to the effects of direct- (e.g.

Corresponding Author: Charles P France, Department of Pharmacology, University of Texas Health Science Center at San Antonio, 7703 Floyd Curl Drive, Mail Code 7764, San Antonio, TX 78229. france@uthscsa.edu; voice 210 567-6969; fax $210567-0104$.

Disclosure/conflict of interest: The authors have no conflict of interest.

The content of this article is solely the responsibility of the authors and does not necessarily represent the official views of the National Institute on Drug Abuse or the National Institutes of Health. 
quinpirole) and indirect-acting (e.g. cocaine) dopamine receptor agonists (Baladi and France 2010; Baladi et al., 2012a,b). Female rats are more sensitive than male rats to the behavioral effects of cocaine (Anker and Carroll, 2011) and eating high fat chow enhances the sensitivity of adult female rats, but not adult male rats, to cocaine-induced locomotion and sensitization (Baladi, unpublished observation). Age-dependent effects of diet on cocaine sensitivity have also been reported (Baladi et al., 2012b) suggesting that adolescent female rats might be more vulnerable than adult female rats, or male rats, to dietary manipulations. It is unclear whether consumption of other preferred substances (e.g. sucrose or saccharin) also increases sensitivity of adolescent female rats to the behavioral effects of cocaine.

Intermittent access to a preferred substance can impact sensitivity to the effects of drugs acting on dopamine systems (Avena and Hoebel, 2003). Although access schedules vary across studies, intermittent feeding schedules often are accompanied by "binge" type intake of sugar or fat (Avena and Hoebel, 2003; Corwin et al., 1998; Puhl et al., 2011). The present experiment investigated whether continuous or intermittent access (i.e. 2 days per week) to high fat chow, sucrose or saccharin increases sensitivity of adolescent female rats to cocaine-induced locomotion and sensitization. This intermittent access protocol was chosen on the basis of a previous study (Cottone et al., 2008) in which 2 days per week of access to a preferred food significantly increased intake of a preferred food.

\section{Methods}

\section{Subjects}

Female Sprague Dawley rats ( $\mathrm{N}=32$; Harlan, Indianapolis, Indiana, USA), weighing 35-49 $\mathrm{g}$ upon arrival, were housed individually in an environmentally controlled room $\left(24 \pm 1^{\circ} \mathrm{C}\right.$, $50 \pm 10 \%$ relative humidity; $12: 12 \mathrm{hr}$ light/dark cycle with lights on $0700-1900 \mathrm{hr}$ ).

Experiments were conducted in accordance with the Institutional Animal Care and Use Committee, the University of Texas Health Science Center at San Antonio, and with the 2011 Guide for Care and Use of Laboratory Animals (Institute of Laboratory Animal Resources on Life Sciences, the National Research Council, and the National Academy of Sciences).

\section{Feeding conditions}

Beginning on postnatal day (PND) 21, subjects had free access to water and standard laboratory chow in the home cage. Beginning on PND 25, rats ( $\mathrm{n}=4-5 /$ group) ate either high fat chow (60\% kcal from fat; Harlan Teklad 06414) and drank water, or standard chow (17\% kcal from fat; Harlan Teklad 7912) and drank either water, $10 \%$ sucrose solution (w/v), or $0.1 \%$ saccharin solution (w/v). Rats either had continuous access to high fat chow, sucrose, or saccharin or they had intermittent access to these substances for 2 days per week with access to water and standard chow on the other 5 days (i.e. not concurrent access). Concentrations of sucrose and saccharin were chosen based on previous studies (Baladi et al., 2011b; Carroll et al., 2007). Intermittent access began at the same time of day (14.00 h) and lasted $48 \mathrm{~h}$. Intermittent access occurred on the same days of the week for an individual subject and tests with cocaine were conducted 3 days after an intermittent access period. 


\section{Locomotor activity}

On PND 22, 23, and 24, rats were placed in locomotor chambers for a 30-min habituation period followed by injections of saline administered every $15 \mathrm{~min}$ for a total of 5 injections (105 min). On 6 occasions (PND 25 [before dietary manipulation], 32, 39, 46, 53 and 60) rats received an injection of saline followed by cumulative doses of cocaine (1.0, 3.2, 10, $17.8 \mathrm{mg} / \mathrm{kg}$; i.p.) administered every $15 \mathrm{~min}$ for a total of 5 injections (105 min).

\section{Data Analyses}

Cocaine-induced locomotor activity was analyzed as a change from locomotor activity after saline (PND 24) using counts during the last 5 min of each 15-min interval. Comparisons were made between feeding conditions (high fat, sucrose, and saccharin compared to standard chow and water) using two-way repeated measures ANOVAs (feeding condition by dose). Sensitization to cocaine-induced locomotion was analyzed by comparing area under the curve (AUC) for each cocaine dose-response curve across weeks using two-way repeated measures ANOVAs (feeding condition by week). Multiple comparisons were made with the Bonferroni test or Holm-Sidaks test where appropriate. For all tests, $p<0.05$ (GraphPad Software Inc., San Diego, California, USA).

\section{Drugs}

Cocaine hydrochloride (NIDA Research Technology Branch, Rockville, MD, USA) was dissolved in sterile $0.9 \%$ saline and administered i.p. in a volume of $1 \mathrm{ml} / \mathrm{kg}$ body weight.

\section{Results}

\section{Body Weight}

Subjects in all groups gained weight throughout the course of the experiment from an average weight of $50.0 \pm 0.2 \mathrm{~g}$ (mean \pm SEM) at PND 21 to $206.3 \pm 2.7 \mathrm{~g}$ at PND 60; however, there was no significant difference in body weight among any of the groups (data not shown).

\section{Feeding Conditions}

Food and fluid intake increased for most rats across the 35 days of this study and varied markedly across some feeding conditions. Eating high fat chow did not markedly alter water intake (panel A, Fig. 1). Rats with continuous access to sucrose (gray squares, panel C) or continuous access to saccharin (gray triangles, panel E) drank more than rats with continuous access to water (gray circles, panels A, C, and E), regardless of whether those drinking water ate high fat or standard chow (Fig. 1). On the 2 days per week that rats in the intermittent groups had access to sucrose or saccharin they drank as much as, or in some cases slightly more than, rats with continuous access to sucrose or saccharin, respectively (compare gray and black squares [panel C] and gray and black triangles [panel E], Fig. 1). On the 5 days per week that rats in the intermittent groups had access to water they drank comparatively less and only as much as rats that drank water throughout the study (compare open squares and gray circles [panel C] and open triangles and gray circles [panel E], Fig. 1). 
Rats with continuous access to high fat chow (gray diamonds, panel B) or sucrose (gray squares, panel D), but not rats with continuous access to saccharin (gray triangles, panel F), ate more than rats eating standard chow and drinking water (gray circles, panels B, D, and F) and those differences increased over the course of the study (Fig. 1). On the 2 days per week that rats in the intermittent groups had access to high fat chow or sucrose they ate as much as rats with continuous access to high fat chow or sucrose, respectively (compare gray and black diamonds [panel B] and gray and black squares [panel D], Fig. 1). On the 5 days per week that rats in the intermittent groups had access to standard chow (panel B) and water (panel D) they ate comparatively more and as much as, in some cases more than, rats that ate standard chow and drank water throughout the study (compare open diamonds and gray circles [panel B] and open squares and gray circles [panel D], Fig. 1). Following 2 days of drinking saccharin in the intermittent group, the recovery of food intake occurred very gradually over the next 5 days of drinking water (open squares, panel D, Fig. 1). The recovery in food intake in rats with intermittent access to high fat chow was more rapid (open diamonds, panel B, Fig. 1). Food intake tended to be increased in rats with intermittent or continuous access to saccharin compared with rats drinking water only (panel F, Fig. 1). Cocaine tests (arrows, Fig. 1) did not appear to systematically alter fluid or food intake.

\section{Locomotor Activity}

Cocaine dose-dependently increased locomotor activity in all groups (Fig. 2, panels A, C, and $\mathrm{E}$ ) but to a significantly greater extent in rats with continuous access to high fat chow (black diamonds, panel A) as compared to rats with continuous access to standard chow and water $(\mathrm{F}[4,39]=4.80 ; p<0.01$; gray circles, panel A). Intermittent access to high fat chow (open diamonds, panel A, Fig. 2), or drinking either sucrose (squares, panel C) or saccharin (triangles, panel E), regardless of access conditions, did not impact cocaine-induced locomotion. Sensitization to cocaine developed faster in rats with continuous access to high fat chow compared to rats with continuous access to standard chow and water $(\mathrm{F}=[5,30]$ 3.612; $p<0.02$; compare black diamonds and gray circles, panel B, Fig. 2). The rate at which sensitization developed was not significantly different among all other groups (Fig. 2, panels $\mathrm{B}, \mathrm{D}$, and F).

\section{Discussion}

Feeding condition can impact the sensitivity of rats to the behavioral effects of drugs acting directly (Baladi et al., 2011a) or indirectly (Baladi et al., 2012b) on dopamine receptors. In the present study the rate and magnitude of sensitization to cocaine-induced locomotion were greatest in adolescent female rats eating high fat chow (see also Baladi et al., 2012b). This study also examined whether intermittent access to high fat chow or (continuous or intermittent) access to sucrose or saccharin similarly enhances sensitivity to the effects of cocaine. In contrast to the effect of continuous access to high fat chow, rats with intermittent access to high fat chow were not more sensitive to cocaine-induced locomotion and they did not develop sensitization more quickly, compared with rats eating standard chow. Drinking sucrose or saccharin (continuous or intermittent access) did not significantly affect cocaineinduced locomotion or the development of sensitization. 
In the current study, intermittent access to high fat chow did not impact sensitivity to the locomotor stimulating effects of cocaine; however, in a previous study intermittent access to high fat chow enhanced sensitivity of rats to the positive reinforcing effects of cocaine (Puhl et al., 2011). While a direct comparison between experiments is limited by the different parameters used (e.g. type of fat, duration of access to diet), rats in the prior study escalated their fat intake ("binged") during the course of the experiment. In the present study, intake of high fat chow was similar between rats with intermittent access and those with continuous access, suggesting that rats with intermittent access did not "binge" on high fat chow; however, rats with intermittent access ate more on days when they had access to high fat chow compared with days when they had access to standard chow. The prior study used male rats while the present study used female rats; thus, differences in results between studies might be related to other well-documented sex differences in sensitivity to behavioral effects of cocaine (Craft and Stratmann, 1996; Chin et al., 2001; Lynch and Carroll, 1999) and to the impact of dietary manipulations on sensitivity to cocaine (Baladi et al., 2012b). Finally, the current study examined the locomotor stimulating effects of cocaine, whereas the previous study (Puhl et al., 2011) examined the reinforcing effects of cocaine using a self-administration procedure; thus, differences in results between studies might also be due to whether the administration of cocaine was response contingent.

Drinking sucrose did not enhance sensitivity to the locomotor effects of cocaine. In a previous study, adult male rats drinking sucrose were more sensitive than adult male rats drinking water to quinpirole-induced yawning (Baladi et al., 2011b); however, sucrose shifted only the ascending (dopamine $\mathrm{D}_{3}$ receptor-mediated) limb of the quinpirole doseresponse curve leftward, while eating high fat chow shifted both the ascending and descending (dopamine $\mathrm{D}_{2}$ receptor-mediated) limbs to the left (Baladi and France, 2010; Baladi et al., 2011a). That sucrose only impacted $\mathrm{D}_{3}$ receptor-mediated behavior, while high fat chow impacted both $D_{3}$ and $D_{2}$ receptor-mediated behavior, might indicate that high fat chow has a broader impact than sucrose on dopamine systems, resulting in a differential effect on sensitivity to drugs acting on these systems. Because the prior study used male rats, the absence of an effect of sucrose in the present study using female rats appears to provide another significant sex-related difference in the behavioral pharmacology of cocaine in rats.

In summary, results of the present study confirm that eating high fat chow dramatically enhances the sensitivity of adolescent female rats to the behavioral effects of cocaine, whereas drinking sucrose or saccharin does not appear to alter sensitivity to cocaine. Together with other published data, these results underscore the fact that the impact of eating condition on the behavioral effects of cocaine varies significantly between sexes and depending on the amount as well as the composition of the diet. That the sensitivity of male and female rats is differentially affected by some dietary manipulations is likely related to previously described sex-related differences in the neurobiology of dopamine systems.

\section{Acknowledgments}

This work was supported, in part, by the National Institutes of Health National Institute on Drug Abuse [Grants K05DA017918 and T32DA031115] 


\section{References}

Anker JJ, Carroll ME. Responding during signaled availability and nonavailability of iv cocaine and food in rats: age and sex differences. Psychopharmacology. 2011; 215:785-799. [PubMed: 21301816]

Avena NM, Hoebel BC. A diet promoting sugar dependency causes behavioral cross-sensitization to a low dose of amphetamine. Neuroscience. 2003; 122:17-20. [PubMed: 14596845]

Baladi MG, France CP. Eating high-fat chow increases the sensitivity of rats to quinpirole-induced discriminative stimulus effects and yawning. Behav Pharmacol. 2010; 21:615-260. [PubMed: 20729718]

Baladi MG, Newman AH, France CP. Influence of body weight and type of chow on the sensitivity of rats to the behavioral effects of the direct-acting dopamine-receptor agonist quinpirole. Psychopharmacology. 2011a; 217:573-585. [PubMed: 21544521]

Baladi MG, Newman AH, Thomas YM, France CP. Drinking sucrose enhances quinpirole-induced yawning in rats. Behav Pharmacol. 2011b; 8:773-778. [PubMed: 21979833]

Baladi MG, Daws LC, France CP. You are what you eat: influence of type and amount of food consumed on central dopamine systems and the behavioral effects of direct- and indirect-acting dopamine receptor agonists. Neuropharmacology. 2012a; 63:76-86. [PubMed: 22710441]

Baladi MG, Koek W, Aumann M, Velasco F, France CP. Eating high fat chow enhances the locomotor-stimulating effects of cocaine in adolescent and adult female rats. Psychopharmacology. 2012b; 222:447-457. [PubMed: 22418731]

Chin J, Sternin O, Wu HB, Fletcher H, Perrotti L1, Jenab S, Quinones-Jenab V. Sex differences in cocaine-induced behavioral sensitization. Cell Mol Biol (Noisy-le-grand). 2001; 47:1089-1095. [PubMed: 11785661]

Carroll ME, Anderson MM, Morgan AD. Higher locomotor response to cocaine in female (vs. male) rats selectively bred for high (HiS) and low (LoS) saccharin intake. Pharmacol Biochem Behav. 2007; 88:94-104. [PubMed: 17707494]

Corwin RL, Wojnicki FHE, Fisher JO, Dimitriou SG, Rice HB, Young MA. Limited access to a dietary fat option affects ingesitve behavior but not body composition in male rats. Physiol Behav. 1998; 65:545-553. [PubMed: 9877422]

Cottone P, Sabino V, Steardo L, Zorrilla EP. Intermittent access to preferred food reduces the reinforcing efficacy of chow in rats. Am J Physiol Regul Integr Comp Physiol. 2008; 294:R10661076. [PubMed: 18667718]

Craft RM, Stratmann JA. Discriminative stimulus effects of cocaine in female versus male rats. Drug Alcohol Depend. 1996; 42:27-37. [PubMed: 8889401]

Gosnell BA. Sucrose intake enhances behavioral sensitization produced by cocaine. Brain Res. 2005; 103:194-201. [PubMed: 15649444]

Lynch WJ, Carroll ME. Sex differences in the acquisition of intravenously self-administered cocaine and heroin in rats. Psychopharmacology. 1999; 144:77-82. [PubMed: 10379627]

Puhl MD, Cason AM, Wojnicki FH, Corwin RL, Grigson PS. A history of bingeing on fat enhances cocaine seeking and taking. Behav Neurosci. 2011; 125:930-942. [PubMed: 21988520] 

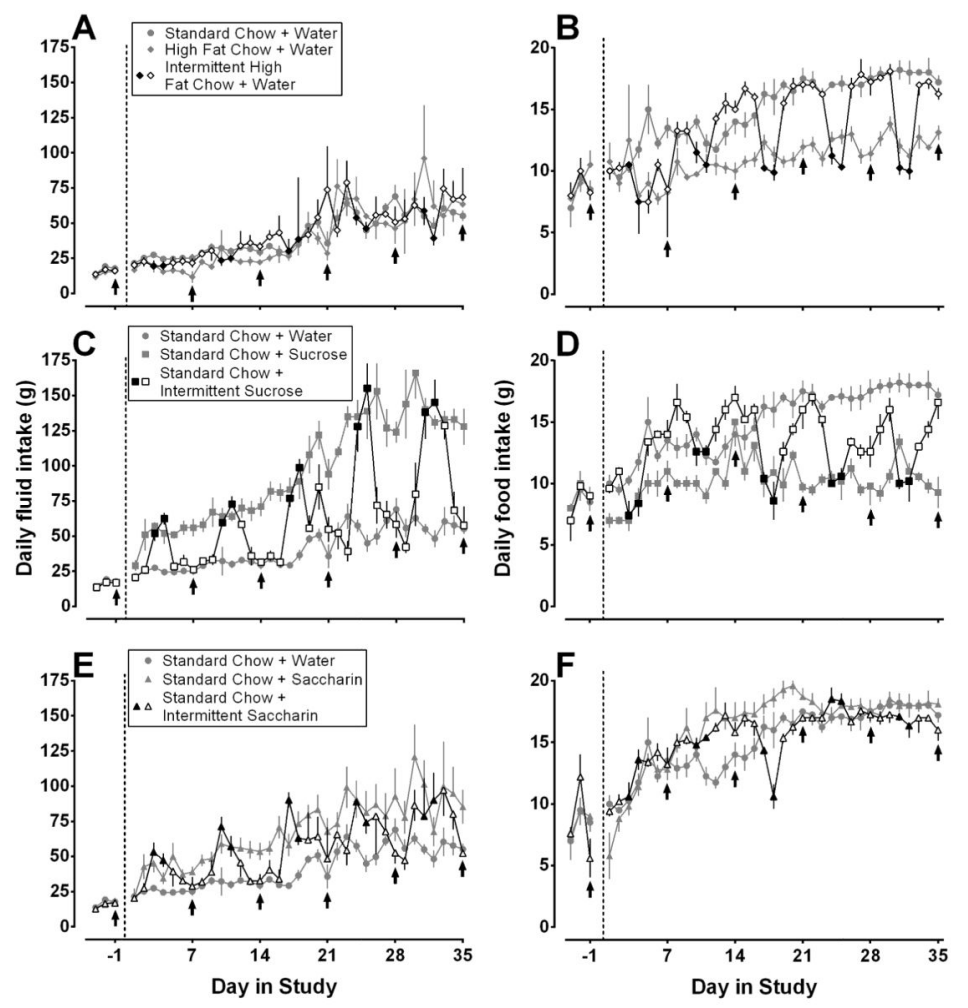

Fig 1.

Mean $( \pm$ SEM) daily fluid (A, C, E) and food (B, D, F) intake throughout the study (g). Data to the left of the vertical dashed lines are from baseline conditions when all rats ate standard chow and drank water. Thereafter (to the right of the dashed lines), rats were assigned to the following feeding conditions: panels $\mathrm{A}$ and $\mathrm{B}$ ) continuous access to water and either continuous access to standard chow (gray circles, $n=4$ ), continuous access to high fat chow (gray diamonds, $n=4$ ), or intermittent access to high fat chow (black [high fat chow 2 days per week] and white [standard chow 5 days per week] diamonds, $n=4)$; panels C and D) continuous access to standard chow and either continuous (gray squares, $n=5$ ) or intermittent (black [sucrose 2 days per week] and white [water 5 days per week] squares, $n=5$ ) access to a $10 \%$ sucrose solution; panels $\mathrm{E}$ and F) continuous access to standard chow and either continuous (gray triangles, $n=5$ ) or intermittent (black [saccharin 2 days per week] and white [water 5 days per week] triangles, $n=5$ ) access to a $0.1 \%$ saccharin solution. Gray circles in panel $\mathrm{A}$ are repeated in panels $\mathrm{C}$ and $\mathrm{E}$ and those in panel $\mathrm{B}$ are repeated in panels $\mathrm{D}$ and $\mathrm{F}$. Cocaine tests are indicated by arrows. Vertical axes: fluid or food consumption in $\mathrm{g}$. Horiontal axes: day in study. 
A
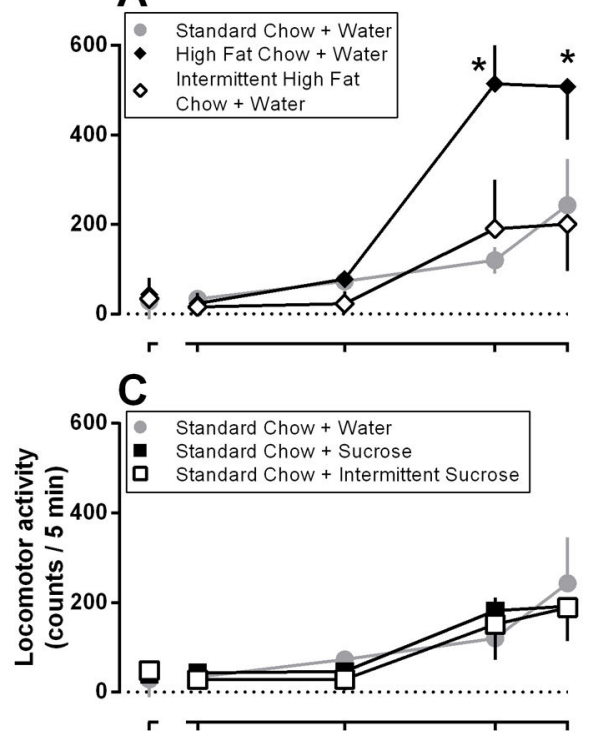

E

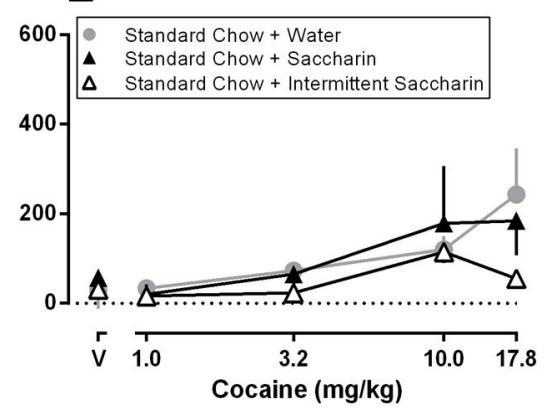

B
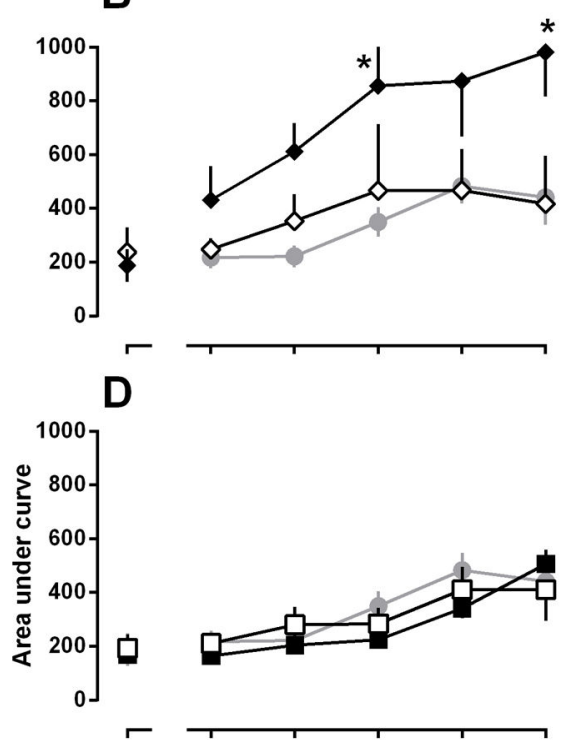

$\mathbf{F}$

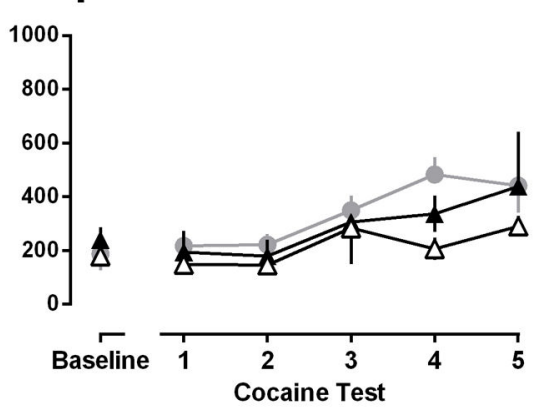

Fig 2.

Locomotor effects of cocaine in rats maintained under different feeding conditions. Left panels (A, C, E): effects of cocaine (1.0-17.8 mg/kg) on the last (PND 60) drug test; data are expressed as the mean $( \pm$ SEM) change from locomotion observed after the administration of saline (PND 24); values are the number of counts in the last 5 min of each 15-min cycle (ordinate); horizontal axes: $\mathrm{V}$ (vehicle) and the dose of cocaine in $\mathrm{mg} / \mathrm{kg}$ body weight. Right panels (B, D, F): effects of cocaine under control conditions (standard chow and water for all rats) and in 5 subsequent tests when rats were maintained on different feeding conditions (see Fig. 1 for details); data are expressed as the average ( \pm SEM) area under the curve (ordinate) for cocaine dose-response curves (1-17.8 mg/kg; abscissa). Gray circles in panel $\mathrm{A}$ are repeated in panels $\mathrm{C}$ and $\mathrm{E}$ and those in panel $\mathrm{B}$ are repeated in panels $\mathrm{D}$ and $\mathrm{F}$. *Denotes significantly different from rats eating standard chow and drinking water. 